Archives de sciences sociales des religions

156 | octobre-décembre 2011

Bulletin Bibliographique

\title{
P. G. Maxwell-Stuart (éd., trad.), The Malleus Maleficarum
}

Manchester -New York, Manchester University Press, 2007, X-266 p.

Jérôme Rousse-Lacordaire

\section{OpenEdition}

Journals

Édition électronique

URL : http://journals.openedition.org/assr/22924

DOI : $10.4000 /$ assr.22924

ISSN : $1777-5825$

Éditeur

Éditions de l'EHESS

Édition imprimée

Date de publication : 31 décembre 2011

Pagination : 224

ISBN : 9782713223273

ISSN : 0335-5985

Référence électronique

Jérôme Rousse-Lacordaire, «P. G. Maxwell-Stuart (éd., trad.), The Malleus Maleficarum », Archives de sciences sociales des religions [En ligne], 156 | octobre-décembre 2011, document 156-79, mis en ligne le 16 février 2012, consulté le 21 septembre 2020. URL : http://journals.openedition.org/assr/22924 DOI : https://doi.org/10.4000/assr.22924

Ce document a été généré automatiquement le 21 septembre 2020

(c) Archives de sciences sociales des religions 


\title{
P. G. Maxwell-Stuart (éd., trad.), The Malleus Maleficarum
}

Manchester -New York, Manchester University Press, 2007, X-266 p.

\author{
Jérôme Rousse-Lacordaire
}

\section{RÉFÉRENCE}

P. G. MAXWELl-StUART (éd., trad.), The Malleus Maleficarum, Manchester -New York, Manchester University Press, 2007, X-266 p.

1 À en croire la quatrième de couverture, cette traduction anglaise de 2007 du Malleus maleficarum serait la première depuis 1928. Malheureusement, cette affirmation est inexacte, car en 2006, Christopher S. Mackay a publié aux Presses de l'Université de Cambridge, en deux volumes, l'édition et la traduction intégrales de cette œuvre, accompagnées d'une introduction de près de deux cents pages et d'un minutieux appareil de notes. Le propos de cette nouvelle traduction anglaise, la troisième donc, est plus modeste : elle ne donne que des extraits de l'ouvrage (les passages omis étant alors résumés), l'apparat critique est réduit et l'introduction est relativement courte (38 p.)

2 Le choix des extraits est judicieux, donnant un bon aperçu des différentes questions traitées et permettant ainsi de se faire rapidement une idée correcte du traité. Les notes sont pertinentes, indiquant aussi bien les sources (notamment bibliques) que les choix du traducteur (dont certains sont discutables, comme par exemple, dans la deuxième question 15 de la troisième partie, la traduction de « ubi sua habent maleficia abscondita " par " where they usually hide their protective amulets", ce que Mackay rend plus exactement par " the situation where they have hidden their devices for sorcery " - plus simplement encore, Armand Danet traduisait par «là où elles cachent leurs maléfices »). On peut cependant se demander pourquoi P.G. Maxwell-Stuart prend pour texte de référence une édition du Malleus maleficarum, celle de Francfort en 1588, 
postérieure de plus d'un siècle à l'édition princeps (Spier, 1486-1487), alors même qu'il existe des fac-similés de cette dernière.

3 Mais, étant donné que le lecteur francophone dispose déjà d'une bonne traduction dans sa langue, celle d'Armand Danet, Le Marteau des sorcières, parue en 1973 chez Plon et depuis lors plusieurs fois rééditée (encore en 2005) chez Jérôme Millon à Grenoble, c'est surtout l'introduction qui retiendra notre attention. Armand Danet introduisait sa traduction par une étude de plus de soixante-dix pages («L'inquisiteur et ses sorcières »); Mackay par une "General introduction », une "Introduction to the latin text " et une "Introduction to the translation", lesquelles formaient un ensemble de près de deux cents pages ; P. G. Maxwell-Stuart, pour sa part, se contente de trente-huit pages, format qui correspond bien à celui de l'ensemble du volume (moins de trois cents pages, alors que l'ouvrage de Danet en comptait près du double, et celui de Mackay plus du quadruple). Cette introduction est divisée en cinq parties : les deux premières (« The intellectual ambience of the Malleus » et "Magic in the fifteenth century ») traitent du contexte culturel dans lequel s'est inscrit le Malleus maleficarum ; la suivante (« Heinrich Institoris and Jakob Sprenger»), des auteurs, présumés ou réels, du Malleus; la quatrième ("The Malleus Maleficarum »), de l'ouvrage en lui-même ; la dernière ("The later history of the Malleus »), de la transmission et de la postérité du Malleus.

Concernant le milieu intellectuel, P.G. Maxwell-Stuart insiste à juste titre sur le sentiment de menace d'hérésie et de division qui régnait alors dans le christianisme occidental, particulièrement chez les clercs. Il souligne ainsi, dans ce contexte, l'importance de caractérisation de la sorcellerie comme hérésie, conception qu'il fait remonter à Nider (probablement au Formicarius et au Praeceptorum divinae Legis, l'un et l'autre des années 1437-1438); toutefois, il faut remarquer que cette assimilation de la sorcellerie à une hérésie, tributaire de l'émergence décisive de la notion de factum heriticale, était déjà présente, quoique contestée, dans la consultation de 1320 super questionibus de baptizacione ymaginibus et aliarum superstitionum (éditée par Alain Boureau, Le Pape et les Sorciers, 2004) et dans la bulle de 1326-1327 Super illius specula de Jean XXII (Alain Boureau, Satan hérétique, 2004). Il relève aussi que, pour nombre de ces derniers, les femmes étaient jugées davantage susceptibles que les hommes d'y succomber, mais, à la suite de l'importante étude de Walter Stephens, (Demon lovers, 2002), il tempère les récurrentes accusations de misogynie portées contre le Malleus en remarquant : que celle-ci y est circonscrite et que les femmes n'y sont pas totalement responsables de leur faiblesse native, à laquelle elles sont cependant capables de résister (pour une période plus tardive, de 1590 à 1631, et dans une zone bien circonscrite, Eichstätt, en Franconie, Jonathan B. Durrant arrive à de semblables conclusions quant à la proportion massive de femmes, $80 \%$, dans les poursuites contre les sorciers: Witchcraft, Gender and Society in Early Modern Germany, 2007). Ce qu'avait d'ailleurs particulièrement montré W. Stephens, c'était que la démonologie savante des $\mathrm{XV}^{\mathrm{e}}$ et $\mathrm{XVI}^{\mathrm{e}}$ siècles avait eu pour moteur déterminant un besoin de croire et de résister au scepticisme. Cet aspect est implicitement souligné par la présentation de la magie au $\mathrm{Xv}^{\mathrm{e}}$ siècle que fait P. G. Maxwell-Stuart. En effet, ce dernier explique que sur la question alors controversée des chevauchées nocturnes et aériennes des sorcières, alors même que le canon Episcopi du Decretum magistri Gratiani, référence obligée en la matière, en niait formellement la possibilité, le Malleus en affirme très nettement la réalité et déclare coupable d'errores hereticales ceux qui prétendraient le contraire (quitte à s'arranger avec le canon Episcopi en prétendant que ce qu'il nie, ce n'est pas la réalité de 
ces chevauchées, mais le fait qu'elles seraient faites en compagnie de Diane et d'Hérodiade: Malleus, pars 1, questio 1, responsio). Cette question resta toutefois encore débattue après la publication du Malleus, ainsi qu'en témoignent la réticence et la prudence des tribunaux ecclésiastiques espagnols à l'accepter trente ans plus tard encore (Gunnar W. Knutsen, Servants of Satan and Masters of Demons, 2009). P. G. Maxwell-Stuart montre ensuite la prégnance dans le Malleus et dans les ouvrages similaires des questions liées à la sexualité, à la fertilité et notamment aux relations sexuelles entre humains et démons, et il rappelle alors l'hypothèse de W. Stephens, que nous évoquions plus haut, selon laquelle cet intérêt traduirait chez les inquisiteurs le souci de contrebalancer leurs propres doutes en prouvant de la sorte, de manière indubitable, la réalité du monde spirituel. Il conclut enfin cette deuxième partie sur la question du « contrat » entre les sorciers et les démons. On ne peut que regretter que ce point soit aussi succinctement traité, car il est au cœur des controverses médiévales savantes sur l'efficacité des signes sensibles sacramentels ou magiques (controverses remarquablement exposées par Irène Rosier-Catach dans La Parole efficace, 2004) ; et, de fait, le Malleus, à la suite de Guillaume d'Auvergne (lui-même tributaire en l'espèce du De doctrina christiana d'Augustin) mais à la différence de Thomas d'Aquin, adhère bien à la théorie contractuelle de la causalité des signes sensibles (Malleus, pars 1, questio 2).

5 La troisième partie présente les personnalités de Heinrich Institoris et Jakob Sprenger ; le premier relativement longuement, et en évitant avec bonheur une approche par trop psychologisante; le second très brièvement et en tenant pour désormais acquis qu'il ne fut pour rien dans la rédaction du Malleus. Mackay a en revanche consacré un long développement au rôle de Sprenger dans la composition du Malleus. Au terme d'une minutieuse analyse des indices internes et externes, il conclut, de manière convaincante, que si Institoris fut bien le principal rédacteur et instigateur du Malleus, Sprenger en a fourni l'armature et le matériau théoriques et conceptuels. La question reste donc ouverte.

6 La quatrième partie de l'introduction décrit la structure et résume le contenu du Malleus, dont le caractère composite (ce que Mackay avait relevé à l'appui de sa thèse de la participation de Sprenger à l'ouvrage) est souligné: mélange de discussions scolastiques (maladroitement qualifiées de "Thomisitic ", même s'il est vrai que la première partie du Malleus est largement dominée par les références à Thomas d'Aquin, comme la seconde l'est par Nider et la troisième par le Directorium inquisitorium d'Eymerich, quelque peu oublié par P. G. Maxwell-Stuart), d'anecdotes et d'exempla, de questions de procédure et de droit. Cette description est toutefois incomplète puisqu'elle ne fait pas état des différentes pièces liminaires, lesquelles permettent pourtant de préciser la nature et la portée du Malleus. Il s'agit de : la bulle de 1484 Summis desiderantes d'Innocent VIII, qui mentionne nommément Institoris et Sprenger ; l'Approbatio et subscripcio doctorum alme universitatis Coloniensis du 19 mai 1487, laquelle, bien qu'elle n'apparaisse qu'à partir de la troisième édition du Malleus (1494), n'est certainement pas un faux et, qui, elle aussi, mentionne Institoris et Sprenger; l'Appologia auctoris in malleum maleficarum, qui, encore, mentionne Institoris et Sprenger, et, surtout, qui s'ouvre par une description proprement apocalyptique du temps, confirmant ainsi les notations faites en ce sens par P. G. Maxwell-Stuart.

7 La dernière partie de l'introduction examine la postérité de l'œuvre. P. G. MaxwellStuart y remet en cause l'influence de l'ouvrage. Il note ainsi que, malgré ses nombreuses éditions jusqu'en 1669, il est cependant peu probable qu'un très grand 
nombre d'exemplaires en aient été produits, d'autant que d'autres traités sur le même sujet, parfois en langue vulgaire, paraissaient et que les lois et règlements gouvernant les procédures des inquisitions locales étaient nombreux, complexes et pas toujours aisés à accorder entre eux. On peut ajouter, avec Danet et Mackay, que l'interruption des rééditions entre 1523 et 1574 semble résulter d'un déplacement de l'intérêt des acteurs de l'inquisition vers d'autres objets que la sorcellerie, c'est-à-dire vers la Réforme. De fait, il semble que le Malleus n'ait effectivement pas suscité de poursuites contre les sorciers, mais, ce qui était d'ailleurs son objet premier, les ait plutôt accompagnées. Plus encore, Danet, à la suite de Joseph Hansen, a souligné que le Malleus en appelait à un relais des tribunaux ecclésiastiques par les juridictions civiles, or, ainsi que le montre l'étude de Jonathan B. Durrant (op. cit.) sur les procès en sorcellerie à Valence et à Barcelone de 1478 à 1700 , c'est précisément là où la justice civile est la plus forte que les poursuites et les condamnations pour sorcellerie sont les plus nombreuses et les plus sévères; en revanche, là où l'inquisition prédomine, les procédures sont plus précautionneuses et aboutissent beaucoup plus rarement à des condamnations pour sorcellerie. Sans que l'on puisse forcément généraliser ce constat à l'ensemble de l'Europe occidentale, la piste vaudrait d'être explorée. Enfin, l'introduction se conclut par une brève évocation de la réception du Malleus, au $\mathrm{Xx}^{\mathrm{e}}$ siècle, particulièrement en monde anglophone avec la première traduction anglaise de Montague Summers, dont il montre à la fois le parti-pris politique (l'assimilation des chasses aux sorcières avec la révolution bolchévique) et les erreurs de traduction - ce pourquoi il était besoin, selon son auteur, de cette nouvelle traduction anglaise.

Dans le cadre de la réception paradoxale du Malleus à la fin du xve et au xvI siècle, un point mériterait peut-être d'être souligné : les éditions italiennes du Malleus sont très localisées (Venise) et tardives (1574-1579) ; or, la première parution du Malleus coïncide presque exactement avec les nouveaux développements de la magie savante dans les milieux de l'humanisme florentin (publication du Pimander ficinien en 1471, des pichiennes Oratio de hominis dignitate et des Conclusiones en 1486, de l'Apologia de Pic en 1487, du De vita coelitus comparanda de Ficin en 1489, vite accompagné, lui aussi d'une Apologia). Mais les réponses à cette promotion d'une magie prétendument naturelle, et qui pourtant ne craignait pas, par bien des aspects, de pénétrer dans le domaine fort suspect de la magie astrale ou de la magie invocatoire, si elles puisaient volontiers aux mêmes sources que celles du Malleus maleficarum (Jérôme Rousse-Lacordaire, Une Controverse sur la magie et la kabbale à la Renaissance, 2010), ne citaient pas, à notre connaissance, ce dernier ouvrage. Peut-être faut-il voir une cause de ce silence, voire de cette ignorance, dans le fait que cette magie est précisément une magie "savante " que ses contradicteurs ont bien du mal à rapporter à la magie « populaire » des sorciers et sorcières que dénoncent tout particulièrement le Malleus : la magie promue, à mots plus ou moins couverts, dans ces milieux érudits échappe largement aux catégories de la démonologie de la fin du Moyen Âge qui sont encore celles du Malleus. On pourrait en dire autant de la magia naturalis vantée, à la même époque, non plus par des platoniciens, mais, cette fois, par des aristotéliciens comme Agostino Nifo, Pietro Pomponazzi ou Giovanni Battista della Porta. En bref, ces transformations dans la théorisation de la magie (et il y en eut d'autres ensuite) n'expliquent-elles pas, elles aussi, les limitations de "l'influence pratique » du Malleus que soulignent justement P. G. Maxwell-Stuart ? On sera donc reconnaissant à ce dernier de nous inviter, par sa publication de larges extraits du Malleus maleficarum, à nous poser ces questions. À d'autres d'y répondre, car il a, quant à lui, largement rempli son objectif: en peu de 
pages, donner à ce texte un accès relativement aisé et complet qu'une légende noire barre encore trop souvent. 